\title{
Correction: Phenotypic spectrum associated with a CRADD founder variant underlying frontotemporal predominant pachygyria in the Finnish population
}

\author{
Daniel L. Polla $\mathbb{1}^{1,2}$ - Elisa Rahikkala ${ }^{3}$ Michaela K. Bode ${ }^{4}$ Tuomo Määttä ${ }^{5}$ Teppo Varilo ${ }^{6}$ Thyrza Loman ${ }^{1}$. \\ Anju K. Philips ${ }^{6} \cdot$ Mitja Kurki $^{7,8,9} \cdot$ Aarno Palotie $^{7,8,9,10,11} \cdot$ Jarmo Körkkö $^{12} \cdot$ Päivi Vieira ${ }^{13} \cdot$ Kristiina Avela $^{14}$. \\ Valérie Jacquemin ${ }^{15} \cdot$ Isabelle Pirson $^{15} \cdot$ Marc Abramowicz $^{15}$ - Arjan P. M. de Brouwer ${ }^{1} \cdot$ Outi Kuismin $^{3,9}$. \\ Hans van Bokhoven ${ }^{1} \cdot$ Irma Järvelä ${ }^{6}$
}

Published online: 10 September 2019

(c) European Society of Human Genetics 2019

\section{Correction to: European Journal of Human Genetics https://doi.org/10.1038/s41431-019-0383-8 Published online 26 March 2019}

Post-publication the authors realised they had accidently omitted Dr. Päivi Vieira's name from the author list. Dr. Vieira from the Clinic for Children and Adolescents, PEDEGO Research Unit and Medical Research Center
Oulu, Oulu University Hospital and University of Oulu, Finland, participated in the collaborative work describing CRADD-founder mutation in the Finnish population. She also provided and interpreted clinical data to Table and Supplements and interpreted the EEG-findings that are present in this manuscript. Dr. Vieira has now been included in the original manuscript.
These authors contributed equally: Daniel L. Polla, Elisa Rahikkala, Michaela K. Bode

These authors jointly supervised this work: Hans van Bokhoven, Irma Järvelä

The original article can be found online at https://doi.org/10.1038/ s41431-019-0383-8.

$\triangle$ Irma Järvelä

irma.jarvela@helsinki.fi

1 Department of Human Genetics, Donders Institute for Brain, Cognition and Behaviour, Radboud University Medical Center, Nijmegen, The Netherlands

2 CAPES Foundation, Ministry of Education of Brazil, Brasília, Brazil

3 Department of Clinical Genetics, PEDEGO Research Unit and Medical Research Center Oulu, Oulu University Hospital and University of Oulu, Oulu, Finland

4 Department of Diagnostic Radiology, Oulu University Hospital and Medical Research Center Oulu, Oulu, Finland

5 Disability Services, Joint Authority for Kainuu, Kainuu, Finland

6 Department of Medical Genetics, University of Helsinki, Helsinki, Finland

7 Psychiatric \& Neurodevelopmental Genetics Unit, Massachusetts General Hospital, Boston, MA, USA
8 The Stanley Center for Psychiatric Research, The Broad Institute of MIT and Harvard, Cambridge, MA, USA

9 Institute for Molecular Medicine Finland (FIMM), University of Helsinki, Helsinki, Finland

10 Analytic and Translational Genetics Unit, Department of Medicine, Massachusetts General Hospital, Boston, MA, USA

11 Department of Neurology, Massachusetts General Hospital, Boston, MA, USA

12 Northern Ostrobothnia Hospital District, Center for Intellectual Disability Care, 90220 Oulu, Finland

13 Clinic for Children and Adolescents, PEDEGO Research Unit and Medical Research Center Oulu, Oulu University Hospital and University of Oulu, Oulu, Finland

14 Department of Clinical Genetics, Helsinki University Hospital, Helsinki, Finland

15 Medical Genetics, IRIBHM, Université Libre de Bruxelles, Brussels, Belgium 